\title{
INVERSE ENERGY CASCADE IN ADVANCED MHD TURBULENCE (THE RNG METHOD)
}

\author{
N. KLEEORIN \\ Department of Mechanical Engineering, Ben-Gurion University \\ of Negev, POB 653, 84105 Beer-Sheva, Israel

\section{ROGACHEVSKII} \\ Racah Institute of Physics, Hebrew University of Jerusalem, \\ 91904 Jerusalem, Israel
}

ABSTRACT. The nonlinear (in terms of the large-scale magnetic field) effect of the modification of the magnetic force by an advanced small-scale magnetohydrodynamic (MHD) turbulence is considered. The phenomenon is due to the generation of magnetic fluctuations at the expense of hydrodynamic pulsations. It results in a decrease of the elasticity of the large-scale magnetic field.

The renormalization group (RNG) method was employed for the investigation of the MHD turbulence at the large magnetic Reynolds number. It was found that the level of the magnetic fluctuations can exceed that obtained from the equipartition assumption due to the inverse energy cascade in advanced MHD turbulence.

This effect can excite an instability of the large-scale magnetic field due to the energy transfer from the small-scale turbulent pulsations. This instability is an example of the inverse energy cascade in advanced MHD turbulence. It may act as a mechanism for the large-scale magnetic ropes formation in the solar convective zone and spiral galaxies.

\section{Introduction}

Investigations of fully developed magnetohydrodynamic (MHD) turbulence is important in view of various cosmic applications. Random motions of a conducting fluid can generate both regular largescale magnetic fields (see, for example, Moffatt 1978, Krause and Rädler 1980, Zeldovich et al. 1983) and magnetic fluctuations (Zeldovich et al. 1990). A source of energy of the regular large-scale magnetic field is the turbulent pulsations. It means, that the energy is transported from the small scales of the turbulent pulsations to the large ones. This process is consequence of helicity ( $\alpha$ - effect) and can 
be considered as an example of an inverse energy cascade in advanced MHD turbulence.

While the dissipation of the energy of the regular magnetic field due to a turbulent magnetic diffusion can be interpreted as a direct energy cascade since the energy is transported from large scales to the small ones. The $\alpha-$ effect and the turbulent magnetic diffusion are linear in the large-scale regular magnetic field.

In this paper an another example of the inverse energy cascade in advanced MHD turbulence is considered. It is nonlinear in the large-scale magnetic field effect of modification of the magnetic force by an advanced small-scale MHD turbulence.

The renormalization group (RNG) method was used for the investigation of the MHD turbulence (see, for example, Moffatt 1981, Kichatinov 1985, Yakhot and Orszag 1986, McComb 1990). The RNG method comprises a change of real turbulence by a medium with effective turbulent transport coefficients. This procedure allows to derive equations for the turbulent transport coefficients: turbulent viscosity, turbulent magnetic diffusion and turbulent magnetic coefficients. The latter determine the contribution of the turbulence in the large-scale regular magnetic force.

\section{The RNG method and turbulent transport coefficients}

Let us consider fully developed MHD turbulence with $R e \gg 1$ and $R m \gg 1$, where $R e=u_{0} l_{0} / \nu_{0}$ is the Reynolds number, $R m=$ $u_{0} l_{0} / \eta_{m}$ is the magnetic Reynolds number, $l_{0}$ is the maximal scale of turbulence, $u_{0}$ is the characteristic turbulent velocity, $\nu_{0}$ is the kinematic viscosity, $\eta_{m}=c^{2} / 4 \pi \sigma$ is the magnetic diffusion, $c$ is the light speed; $\sigma$ is the electrical conductivity of the fluid. Let the dissipation due to the molecular viscosities $\nu_{0}$ and $\eta_{m}$ is intrinsic only in the region $l \leq l_{d}$, where $l_{d} \ll l_{0}$.

Numerous works on turbulence are confined of a study of the large-scale properties of flows by averaging the equations over the pulsations of all scales of the turbulence (see, for example, Monin and Yaglom 1975, McComb 1990). While the averaging in the RNG method is performed up to the scale inside the inertial interval of the turbulence. So the turbulent transport coefficients depend on the scale of the averaging. Next step of the RNG method consists in a step by step increasing of the scale of the averaging. This procedure 
allows to derive equations for the turbulent transport coefficients. The RNG method for this problem requires to find an equation invariant under the renormalization of the turbulent transport coefficients. The recent results (Kleeorin et al. 1990) with a simple model for the high order closure procedure were used for deriving the equation.

Let the averaging over the small-scale region of the spectrum of the turbulent pulsations up to the scale $l_{*}$ is performed. The averaged equations for velocity $\mathbf{v}$ and magnetic fields $\mathbf{H}$ have the form

$$
\begin{aligned}
\rho\left(\frac{\partial \mathbf{v}}{\partial t}+(\mathbf{v} \cdot \nabla) \mathbf{v}\right) & =-\nabla\left(p+Q_{p} \frac{H^{2}}{8 \pi}\right)+\frac{Q_{s}}{4 \pi}(\mathbf{H} \cdot \nabla) \mathbf{H}+\nu \Delta \mathbf{v}+\mathbf{f} \\
\frac{\partial \mathbf{H}}{\partial t} & =\operatorname{curl}(\mathbf{v} \times \mathbf{H}-\eta \operatorname{curl} \mathbf{H}+c \mathbf{E}),
\end{aligned}
$$

where $\operatorname{div} \mathbf{v}=0, p$ is the pressure, $\mathbf{f}$ is the external force, $\mathbf{E}$ is the external electric field. The turbulent coefficients $\nu, \eta, Q_{p}$ and $Q_{s}$ depend on the scale of averaging $l_{*}$. If $l_{*}$ tends to the dissipation scale $l_{d}$, the functions $\nu$ and $\eta$ reach the molecular magnitudes $\nu_{0}$, $\eta_{m}$, and the magnetic coefficients $Q_{p}, Q_{s} \rightarrow 1$. The latter determine the contribution of the turbulence in the large-scale regular magnetic force (Kleeorin et al. 1990).

Now let us change the scale of the averaging on a small value $|\Delta \mathbf{k}| \ll k_{*}$, where the wave number $k_{*}=l_{*}^{-1}$. After that we carry out the reaveraging Eqs. (1)-(2) over the turbulent pulsations. Now in the region $k<k_{*}-|\Delta \mathbf{k}|$ the fields $\mathbf{V}$ and $\mathbf{B}$ are regular ones. An region $k>k_{*}-|\Delta \mathbf{k}|$ corresponds to the turbulent pulsations. Because of Eqs. (1)-(2) have been already averaged up to the scale $l_{*}=k_{*}^{-1}$, it is enough to average the equations over pulsations of the velocity $\mathbf{u}$ and the magnetic field $\mathbf{h}$ located in the small region $k_{*}-|\Delta \mathbf{k}|<|\mathbf{k}|<k_{*}$. Here $\mathbf{v}=\mathbf{V}+\mathbf{u}, \mathbf{H}=\mathbf{B}+\mathbf{h}, \mathbf{V}=\langle\mathbf{v}\rangle$ , $\mathbf{B}=\langle\mathbf{H}\rangle$, and the angle brackets denote averaging over the ensemble of turbulent pulsations in the region $k_{*}-|\Delta \mathbf{k}|<|\mathbf{k}|<k_{*}$. Then the equations for the 'mean' fields $\mathbf{V}$ and $\mathbf{B}$ are

$$
\rho\left(\frac{\partial V_{j}}{\partial t}+(\mathbf{V} \cdot \nabla) V_{j}\right)+\frac{\partial}{\partial x_{j}}\left(p+Q_{p} \frac{B^{2}}{8 \pi}\right)-\frac{Q_{s}}{4 \pi}(\mathbf{B} \cdot \nabla) B_{j}-
$$




$$
\begin{gathered}
-\nu \Delta V_{j}-f_{j}=\frac{\partial}{\partial x_{j}}\left(\delta \sigma_{i j}\right) \\
\frac{\partial B_{j}}{\partial t}-\operatorname{curl}_{j}(\mathbf{V} \times \mathbf{B}-\eta c u r l \mathbf{B}+c \mathbf{E})=\operatorname{curl}_{j}<\mathbf{u} \times \mathbf{h}>
\end{gathered}
$$

where the generalized Maxwell-stress tensor (including the Reynolds turbulent -stress tensor) is

$$
\delta \sigma_{i j}=-Q_{p} \frac{<h^{2}>}{8 \pi} \delta_{i j}+\frac{Q_{s}}{4 \pi}<h_{i} h_{j}>-\rho<u_{i} u_{j}>
$$

$\delta_{i j}$ is the Kronecker delta. To obtain a closed system of equations it is important to find the dependence of the second moments $\left\langle h_{i} h_{j}\right\rangle$ ,$<u_{i} u_{j}>$ and $\left\langle u_{i} h_{j}>\right.$ on the 'mean' fields $\mathbf{V}$ and $\mathbf{B}$. To this end substracting Eq. (3) from Eq. (1) and Eq. (4) from Eq. (2), and transforming to the $k$ - and $\Omega$ - spaces, one can get the equations for the turbulent fields. After that it is possible to determine the second moments.

Let us make a comment. Here we introduce the background MHD turbulence. It is the turbulence without the regular large-scale fields ( $\mathbf{V}=0$ and $\mathbf{B}=0$ ). The goal of this paper is a study of the shift from the background turbulence level due to the the presence of the regular large-scale fields. It is important for the investigation of the interaction of the large-scale magnetic field with the turbulence. Thus, the background MHD turbulence is supposed to be given. The background MHD turbulence is equivalent to an introduction of effective external forces. These external fields are called stirring forces in the RNG method (see, for example, McComb 1990).

We conșider quadratic (in terms of the large-scale magnetic field) effects. It means, that $B^{2} / 8 \pi \ll<\rho u^{2}>/ 2$. Finding the second moments one gets the expression for the generalized Maxwell-stress tensor and effective electric field (Kleeorin and Rogachevskii 1992)

$$
(\delta \sigma)_{m n}=\rho\left(\frac{\partial V_{n}}{\partial R_{m}}+\frac{\partial V_{m}}{\partial R_{n}}\right) \Delta \nu-\frac{B^{2}}{8 \pi} \Delta Q_{p} \delta_{m n}+\frac{B_{m} B_{n}}{4 \pi} \Delta Q_{s}
$$




$$
c \delta \mathbf{E}=\operatorname{curl}<\mathbf{u} \times \mathbf{h}>=-\Delta \eta \operatorname{curl} \mathbf{B} .
$$

Here the change of the turbulent coefficients depends on $\Delta k$. Substitution expressions (6)-(7) in Eqs. (3)-(4) shows that the form of these equations is coincide with Eqs. (1)-(2). It means, that these equations are invariant under the procedure of the reaveraging. Passed to the limit $\Delta k \rightarrow 0$, one can get the equations for the turbulent viscosity, turbulent magnetic diffusion and turbulent magnetic coefficients

$$
\begin{gathered}
\frac{d \nu}{d k}=-\frac{7}{30 \nu k^{2}}\left(W_{0}(k)+\frac{5 Q_{s}}{14 \pi \rho} P m M_{0}(k)\right) \\
\frac{d \eta}{d k}=-\frac{2}{3} \frac{P m}{\nu k^{2}(1+P m)}\left(W_{0}(k)+\frac{Q_{s}}{8 \pi \rho} M_{0}(k)\right) \\
\frac{d Q_{s}}{d k}=\frac{Q_{s}}{15} \frac{P m(2+P m)}{\nu^{2} k^{2}(1+P m)}\left(W_{0}(k)-\frac{Q_{s}}{4 \pi \rho}\left(\frac{1+2 P m}{2+P m}\right) M_{0}(k)\right), \\
\frac{d Q_{p}}{d k}=4 \frac{d Q_{s}}{d k}-\frac{Q_{p}}{3} \frac{P m}{\nu^{2} k^{2}(1+P m)}\left(W_{0}(k)-\frac{Q_{s}}{2 \pi \rho} M_{0}(k)\right) .
\end{gathered}
$$

Here $\operatorname{Pm}(k)=\nu(k) / \eta(k)=R m / R e$ is the effective magnetic Prandtl number, $W_{0}(k)$ and $M_{0}(k)$ are spectra of the hydrodynamic and magnetic pulsations of the background MHD turbulence.

It is seen from Eq. (10) that a state in which

$$
W_{0}(k)=\frac{Q_{s}}{4 \pi \rho}\left(\frac{1+2 P m}{2+P m}\right) M_{0}(k)
$$

is special. In this case $Q_{s}(k)=$ const $=1$ and

$$
Q_{p}(k)=\exp \left(-\int_{k_{d}}^{k} \frac{W_{0}(k) P m^{2}}{(\nu k)^{2}(1+P m)(1+2 P m)} d k\right)>0 .
$$

It means, that the effective magnetic pressure $P=Q_{p}\left(B^{2} / 8 \pi\right)$ is always positive. Eq. (12) corresponds to the 'effective' energy equipartition. Note, that the 'usual' equipartition state is $W_{0}(k)=M_{0}(k) / 4 \pi \rho$ 
- Comparison of the expression and (12) shows that in the case of $Q_{s}<1$ and $P_{m}<1$ a level of the magnetic fluctuations can exceed that obtained from the 'usual' equipartition assumption.

We choose spectrum of the background turbulence on the basis of the results of numerical simulations (Meneguzzi et al. 1981) confirmed by analitical estimates (Kleeorin et al. 1986). In the model there are magnetic fluctuations $M_{0}(k)$ of the background turbulence in the small scales $k>k_{m} \simeq \sqrt{R m} k_{0}$, where $k_{0}=l_{0}^{-1}$ - They are determined by (12). While for $k<k_{m} M_{0}(k)=0$ and the spectrum of the hydrodynamic pulsations in this region is $W_{0}(k)=(\beta-1)\left(u_{0}^{2} / k_{0}\right) \cdot\left(k / k_{0}\right)^{-\beta}$. Here $u_{0}$ is the characteristic turbulent velocity in the scale $l_{0}$. Solution of the system (8)-(11) describes a dependence of the turbulent transport coefficients on $k$. For $k=k_{0}$ they determine the large-scale effects and have the form

$$
\nu^{*} \simeq \nu_{T}, \quad \eta^{*} \simeq 1.3 \nu_{T}, \quad Q_{s}^{*} \simeq R m^{-3 \beta / 14}, \quad Q_{p}^{*} \simeq-R m^{\beta / 7}
$$

where $\nu_{T}=\left(u_{0} / k_{0}\right) \sqrt{7(\beta-1) / 30(\beta+1)}$. From equations for the second moments one can get the level of the magnetic fluctuations in the present of the large-scale regular magnetic field (Kleeorin and Rogachevskii 1992). It is

$$
<h^{2}>\simeq<h_{0}^{2}>+\ln (R m) \cdot B^{2} .
$$

This result is in agreement with one got after application of the high order closure procedure (Kleeorin et al. 1990). It is confirmed also by numerical simulation by Brandenburg et al. 1992.

From (14) it is seen that the elasticity of the large-scale magnetic field decrease and the 'effective' magnetic pressure $P=Q_{p}\left(B^{2} / 8 \pi\right)$ changes the sign. This phenomenon results in an excitation of a largescale MHD instability. It leads to formation of inhomogeneities of the regular magnetic field on account of the energy transported from the small-scale turbulent pulsations. This instability is an example of the inverse energy cascade in MHD turbulence. It may act as a mechanism for the large-scale magnetic ropes formation in the solar convective zone and spiral galaxies (Kleeorin et al. 1990, Kleeorin and Rogachevskii 1990). 


\section{REFERENCES}

A. Brandenburg et al. (1992), Astrophys. J., submitted.

N. Kleeorin and I. Rogachevskii, in Proceedings of the ESA Workshop on 'Plasma Astrophysics', Telavi, Georgia (ESA Publ. Div., ESTEC, Dordrecht, 1990), p. 21.

N. Kleeorin and I. Rogachevskii (1992). Phys. Rev. A, submitted.

N. Kleeorin, I. Rogachevskii, and A. Ruzmaikin, Sov. Astron. Lett., 15, 274 (1989).

N. Kleeorin, I. Rogachevskii, and A. Ruzmaikin, Sov. Phys. JETP 70, 878 (1990).

N. Kleeorin, A. Ruzmaikin, and D.D. Sokoloff, in Proceedings of the ESA Workshop on 'Plasma Astrophysics', Sukhumi, Georgia ( ESA Publ. Div., ESTEC, Dordrecht, 1986), p. 557.

L.L. Kichatinov, Magnetohydrodynamics, N 2, 3 (1985).

F. Krause, and K.H. Rädler, Mean-Field Magnetohydrodynamics and Dynamo Theory (Pergamon, Oxford, 1980).

W.D. McComb, The Physics of Fluid Turbulence (Clarendon, Oxford, 1990).

M. Meneguzzi, U. Frisch, and A. Pouquet, Phys. Rev. Lett., 47, 1060 (1981).

H.K. Moffatt, Magnetic Field Generation in Electrically Conducting Fluids (Cambridge Univ. Press, Cambridge, 1978).

H.K. Moffatt, J. Fluid Mech., 106, 27 (1981).

A.S. Monin and A.M. Yaglom, Statistical Fluid Mechanics (MIT Press Cambridge, Massachusetts, 1975), Vol. 2.

V. Yakhot and S.A. Orszag, J. Sci. Comput., 1, 3 (1986).

Ya.B. Zeldovich, A.A. Ruzmaikin, and D.D. Sokoloff, Magnetic Fields in Astrophysics (Gordon and Breach, New York, 1983).

Ya.B. Zeldovich, A.A. Ruzmaikin, and D.D. Sokoloff, The Almighty Chance (Word Scientific Publ., London, 1990). 